\title{
Highly Efficient Antimicrobial and Antifouling Surface Coatings with Triclosan-Loaded Nanogels
}

\author{
Damla Keskin, Lisa Tromp, Olga Mergel, Guangyue Zu, Eliza Warszawik, Henny C. van der Mei, \\ and Patrick van Rijn*
}

Cite This: ACS Appl. Mater. Interfaces 2020, 12, 57721-57731

Read Online



ABSTRACT: Multifunctional nanogel coatings provide a promising antimicrobial strategy against biomedical implant-associated infections. Nanogels can create a hydrated surface layer to promote antifouling properties effectively. Further modification of nanogels with quaternary ammonium compounds (QACs) potentiates antimicrobial activity owing to their positive charges along with the presence of a membrane-intercalating alkyl chain. This study effectively demonstrates that poly(N-isopropylacrylamide-co$\mathrm{N}$-[3(dimethylamino)propyl]methacrylamide) (P(NIPAM-co-DMAPMA)-based nanogel coatings possess antifouling behavior against $S$. aureus ATCC 12600, a Gram-positive bacterium. Through the tertiary amine in the DMAPMA comonomer, nanogels are quaternized with a 1-bromo-dodecane chain via an $\mathrm{N}$-alkylation reaction. The alkylation introduces the antibacterial activity due to the bacterial membrane binding and the intercalating ability of the aliphatic QAC. Subsequently, the quaternized nanogels enable the formation of intraparticle hydrophobic domains because of intraparticle hydrophobic interactions of the aliphatic chains allowing for Triclosan incorporation. The coating with Triclosan-loaded nanogels shows a killing efficacy of up to $99.99 \%$ of adhering bacteria on the surface compared to nonquaternized nanogel coatings while still possessing an antifouling activity. This powerful multifunctional coating for combating biomaterial-associated infection is envisioned to greatly impact the design approaches for future clinically applied coatings.

KEYWORDS: Nanogels, bacterial adhesion, killing efficacy, drug delivery, quaternization

\section{INTRODUCTION}

Biofilm formation is one of the major complications with implants as implant surfaces create a high risk of infection and inflammation. ${ }^{1-3}$ Initial bacterial adhesion is often followed by the growth of microbial communities and extracellular polymeric substance formation, resulting in biofilm formation on the surface. ${ }^{4,5}$ Almost $80 \%$ of the medical associated infections are predicted to originate from biofilm formation. ${ }^{6}$ Many studies are concerned with the treatment of biofilms to kill the bacteria on the implant surfaces only by antibiotics. However, this antibiotic treatment strategy presents a massive disadvantage, due to limited efficacy based on bacterial resistance in the formed biofilms as well as posing difficulty of reaching the target infection. ${ }^{8,9}$ In this sense, alternative approaches such as a combination of antifouling and antimicrobial coatings created using hydrogels may become a more promising strategy. ${ }^{10}$ In such surface modification approaches, the role of antifouling or antimicrobial coatings would be preventing initial bacterial adhesion and formation of a biofilm and meanwhile stopping bacterial growth contamination on the surface.

In the last decade, surface coatings created by nanogels from natural and synthetic hydrophilic monomers have attracted

Received: October 9, 2020

Accepted: December 3, 2020

Published: December 15, 2020 
Scheme 1. Schematic Representation of Antifouling and Antimicrobial Nanogel Coatings

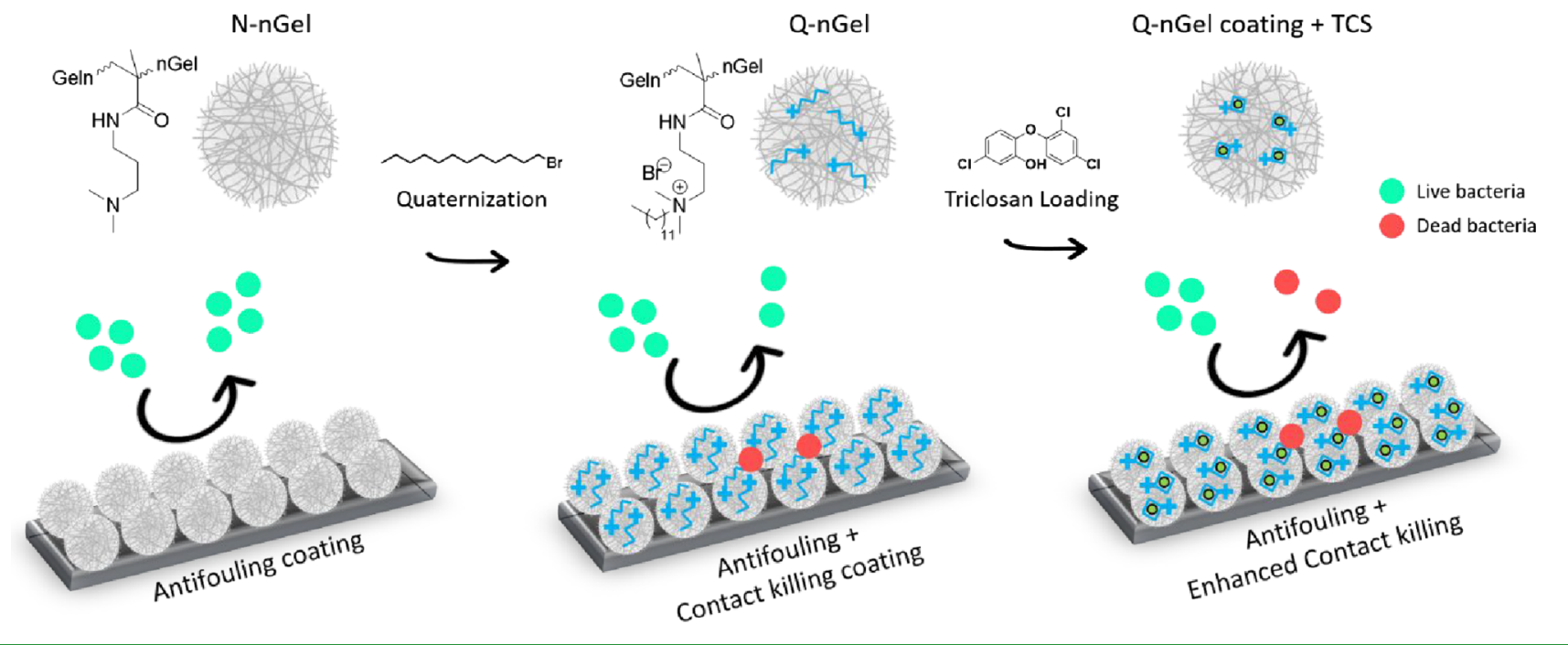

great scientific interest ${ }^{11-15}$ for biomedical applications since they offer an excellent biocompatible environment. ${ }^{16,17}$ Nanogel coatings exhibit a combination of properties from polymer brushes and hydrogel networks, such as robustness, easily tunable composition, and responsiveness. ${ }^{18,19}$ Various applications of nanogel coatings have been described as components for biointerfaces; for instance, nanogel coatings can provide a hydration layer on the surface to prevent fouling. ${ }^{20-22}$ Recently, our group reported the effect of nanogel size, mechanical properties, and coating thickness on the antifouling behavior toward bacterial adhesion. ${ }^{13}$ So far, nanogel coatings have been used for antifouling purposes toward proteins, ${ }^{23}$ macrophage adhesion, ${ }^{24}$ antifogging and antifrosting performance, ${ }^{25}$ enzyme uptake capability for biosensor design, ${ }^{26}$ controlling the cell proliferation and cell adhesion, ${ }^{27,28}$ and controlling antimicrobial and anti-inflammatory properties via peptide-loading. ${ }^{29}$ The surface coating preparation with nanogel particles is a facile method and predominantly based on the electrostatic interactions of a pretreated surface and the nanogel surface charge that is induced by the synthesis method. ${ }^{30,31}$

A crucial advantage of polymeric nanogels and hydrogels is the possibility to have access to a broad range of chemical functional groups to tailor the coatings and introduce antimicrobial properties for contact killing activity or release of antimicrobials in a controlled fashion. ${ }^{32,33}$ Quaternary ammonium compounds (QACs) are well-known for their antibacterial properties and have been covalently bound to surfaces to introduce contact killing. Although the exact killing mechanism of QACs is still not completely understood, it is accepted that positively charged surfaces with quaternaryammonium functionalities kill attached bacteria by disrupting the membrane when the contact occurs between the negatively charged bacterial membrane and positively charged QACs. ${ }^{34-36}$ Recently, we have introduced QACs with long alkyl chains into nanogels to induce killing of bacteria in suspension, ${ }^{37}$ which was more effective than using previously designed quaternization approaches using methyl iodide. ${ }^{25}$ Upon introducing the longer alkyl chain, we found that a more sophisticated killing effect was achieved due to the formation of intraparticle micelles, which facilitated the storage of Triclosan, a hydrophobic antimicrobial that only was released when coming into contact with bacteria and displayed a synergistic killing effect. Although the strategy was ideal for suspension killing approaches, when combating implantassociated bacterial infection, applying it as a coating provides a better chance to prevent infection and is therefore considered as an essential strategy.

Herein, our aim was to transform the suspension killing approach of functionalized nanogels into a multifunctional coating to be able to combat implant-associated infections. To that end, we show the effect of the Triclosan-loaded antifouling nanogel coatings on the antibacterial performance. The nonquaternized nanogel $(\mathrm{N}-\mathrm{nGel})$ with poly $(\mathrm{N}$-isopropylacrylamide-co-N-[3-(dimethylamino)propyl]methacrylamide) (P(NIPAM-co-DMAPMA) has been synthesized and used for the preparation of surface coatings to reveal the antifouling property. Subsequently, these nanogels have been quaternized by $\mathbf{N}$-alkylation with 1-bromo-dodecane (Q-nGel) and applied as a coating not only to have antimicrobial activity on the surface but also to obtain a platform for further encapsulation and active release of Triclosan when bacteria are present and attach as we previously found in suspension. For this purpose, Triclosan has been successfully loaded into the hydrophobic cavity of the Q-nGels on the surface coating to enhance the antimicrobial efficacy (Q-nGel coating+TCS). The resulting nanogel coatings have been tested under flow conditions, and the influence of the nanogel quaternization and Triclosan encapsulation on the antifouling and antibacterial properties has been assessed. With this approach, the Triclosan-loaded coatings owing to the dodecane chain of the quaternized nanogels provide a straightforward and novel antimicrobial approach with multifunctionality composed of both bacterial repelling and bacterial killing capabilities (Scheme 1).

\section{RESULTS AND DISCUSSION}

Characterization of Nanogels. The synthesis of the nonquaternized P(NIPAM-co-DMAPMA) nanogel (N-nGel) was done via precipitation copolymerization with the monomers NIPAM and DMAPMA, cross-linker $N, N^{\prime}$ methylene-bis (acrylamide) (BIS), and 2,2'-azobis(2methylpropionamidine)dihydrochloride (AMPA, V50) as a cationic initiator. Afterward, the tertiary amine of these $\mathrm{N}$ nGels was quaternized using 1-bromo-dodecane (Q-nGel) for 


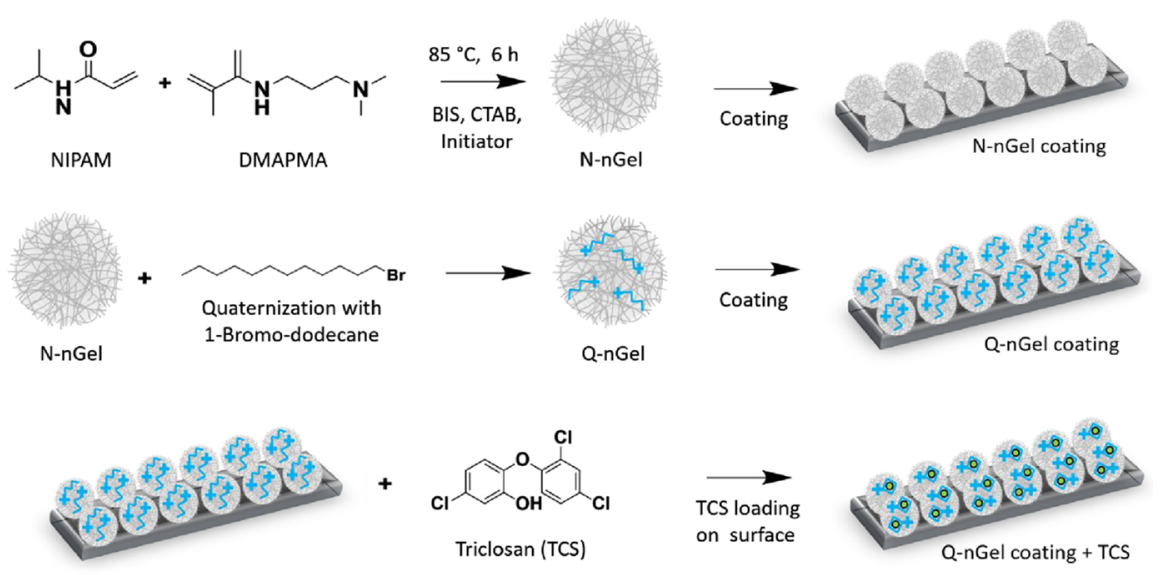

Figure 1. Schematic overview of nanogel formation and preparation of the antifouling and antimicrobial nanogel coating. The synthesis of the nonquaternized nanogel ( $\mathrm{N}$-nGel) was done via precipitation polymerization using NIPAM and DMAPMA. N-alkylation of N-nGel's tertiary amine groups with 1-bromo-dodecane resulted in the quaternized nanogels (Q-nGel). Next, the surface coating on preactivated glass was performed with these nanogels (Q-nGel coating). Finally, the Triclosan-loaded nanogel coating (Q-nGel coating+TCS)) was prepared by incorporating Triclosan into the hydrophobic domains of the intraparticle micelles of the Q-nGel.
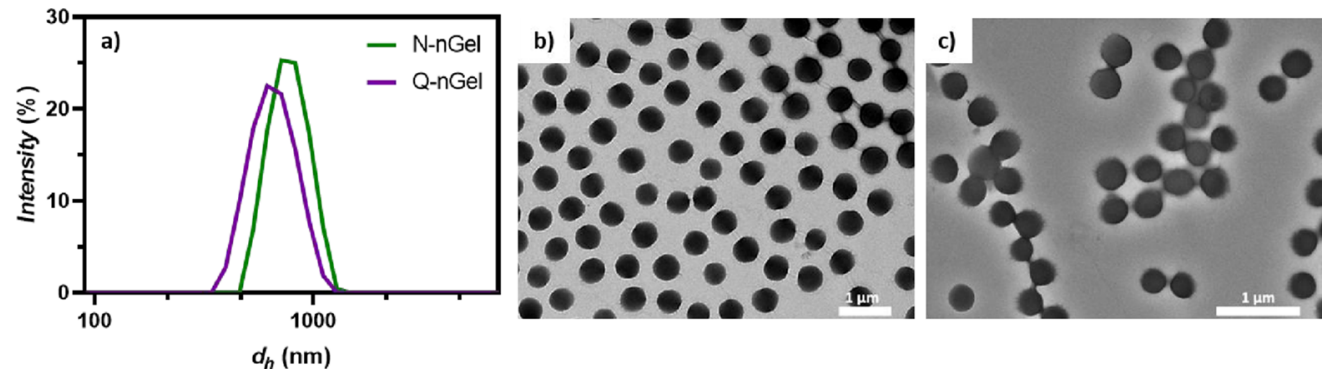

Figure 2. (a) Hydrodynamic diameters $d_{h}$ of nanogels at $24{ }^{\circ} \mathrm{C}$; transmission electron microscopy (TEM) images of (b) N-nGels and (c) Q-nGels in the dry state.


Figure 3. Nanogel-coated glass surfaces at room temperature in the dry state imaged by atomic force microscopy (AFM). (a) N-nGel coating, (b) Q-nGel coating, and (c) Q-nGel coating+TCS.

obtaining the antimicrobial property, as shown in Figure 1 and Figure S1.

The quaternization of the nanogel with 1-bromo-dodecane was proven by ${ }^{1} \mathrm{H}$ NMR analysis (Figure S2). The signal assigned to the dodecane chain appeared at 1.32 and $0.91 \mathrm{ppm}$ in the spectra of Q-nGel, and the signal at $2.3 \mathrm{ppm}$ disappeared, which depicts the methyl protons of DMAPMA, indicating the quaternization. The degree of quaternization is approximately $93 \%$ as determined by ${ }^{1} \mathrm{H}$ NMR analysis of the nanogels upon quaternization in a quantitative fashion.
Dynamic light scattering (DLS) results showed average hydrodynamic diameters (at $24{ }^{\circ} \mathrm{C}$ ) of $763 \pm 7$ and $622 \pm 11$ $\mathrm{nm}$ for $\mathrm{N}$-nGel and Q-nGel, respectively (Figure 2a). The hydrophobic interactions within the nanogel due to the aliphatic chains induce a contraction within the hydrogel network and decrease the diameter upon quaternization. According to transmission electron microscopy (TEM) results, both nanogels possessed spherical shapes with an average diameter of $485 \pm 38 \mathrm{~nm}$ for the $\mathrm{N}-\mathrm{nGel}$ and a smaller value of $311 \pm 29 \mathrm{~nm}$ for Q-nGel (Figure 2b,c). The size difference between these two techniques is ascribed to the solvated state 
of the nanogel particles when measured by DLS, while with TEM, the dry state is visualized.

The thermoresponsive behavior of the N-nGels and Q-nGels was investigated by DLS in ultrapure water (Figure S3). Upon increasing the temperature beyond the volume phase transition temperature (VPTT), the nanogel hydrodynamic radius $\left(R_{\mathrm{h}}\right)$ decreased, which is common for PNIPAM-nanogels. The data show that there is a shift in the VPTT of N-nGel to a temperature range of $35-50{ }^{\circ} \mathrm{C}$, which is higher than the VPTT of $32{ }^{\circ} \mathrm{C}$ that is generally found for conventional poly ( $N$-isopropylacrylamide) (PNIPAM) ${ }^{38}$ The increase of the VPTT is attributed to the charges introduced by DMAPMA. The introduced electrostatic repulsion of the charged DMAPMA comonomer leads to the altered thermosensitivity, which then affects the $R_{\mathrm{h}}{ }^{26}$ Additionally, the protonated DMAPMA amino segments are hydrophilic, leading to a stronger coordination of water molecules, and consequently, the VPTT is affected and the VPTT increase as compared to pure PNIPAM and the decrease in the hydrodynamic radius happen over a broader temperature range. ${ }^{39}$ After quaternization with 1-bromo-dodecane, the thermoresponsiveness was altered compared to the N-nGel most likely because of the already collapsed network due to the intraparticle hydrophobic interactions of the dodecane chains that limit the swelling/deswelling process. As expected, the hydrophobic interactions and permanent charges affect the VPTT after quaternization, which additionally confirms that the modification with 1-bromo-dodecane is successful.

Furthermore, the zeta potential measured in ultrapure water of the protonated $\mathrm{N}-\mathrm{nGel}$ is $+25.2 \pm 1.0 \mathrm{mV}$ and the Q-nGel $+33.6 \pm 1.1 \mathrm{mV}$, which supports that the quaternization increased the zeta potential.

Nanogel Coating Formation and Loading Strategy. Nanogel Coating Formation. The synthesized nanogel particles were immobilized on a glass surface to create the nanogel coating. Figure 3 shows the AFM images of the NnGel coating, Q-nGel coating, and the Q-nGel coating+TCS on glass surfaces. The positively charged $\mathrm{N}$-nGels were sprayed on the glass slide after surface activation with plasma oxidation. After the washing step, the resulting homogeneous nanogel coating displayed a surface coverage of more than $90 \%$ (Figure 3a). The same method was applied for Q-nGel coating preparation. However, the adsorption of Q-nGel onto the plasma-treated glass surface was much lower than that of $\mathrm{N}$ nGel (Figure S4a); hence, a similar surface coverage could not be obtained. This can be attributed to the strong ionic interparticle repulsion among Q-nGels and weak interaction between nanogels and the plasma-treated glass surface, as shown before. $^{31,40,41}$ To obtain a fully packed surface, a solution of poly(sodium 4-styrene sulfonate) (PSS) was used as a polyanionic anchoring layer to enhance the electrostatic attractions between Q-nGels and the surface. Thus, following the plasma oxidation, the deposition of hyperbranched polyethylenimine (PEI) was performed, and then, PSS is adsorbed on the glass surface (Figure S4b). Subsequently, QnGel solution was sprayed onto the PSS-coated glass, resulting in a close-packed nanogel layer on the surface as expected (Figure 3b).

Loading Strategy. Due to the aliphatic tails attached to the Q-nGel network, the surface-bound Q-nGels can be loaded with Triclosan to further increase the antimicrobial effectiveness of the coating. The loading of Triclosan into the hydrophobic cavities is possible due to the intraparticle micelle formation of the introduced dodecane chains in the aqueous environment. ${ }^{42,43}$ These intraparticle micelles facilitate the encapsulation of hydrophobic components such as Triclosan, as shown in suspension in previous research. ${ }^{37}$ To achieve the Triclosan incorporation on the coated surface, a previously prepared Q-nGel bound glass substrate is immersed in a Triclosan solution. Figure $3 \mathrm{c}$ shows the coating pattern with a high surface coverage of the nanogel layer after the Triclosan loading.

Additionally, nanogel coatings exhibit good stability after washing with mechanical agitation following the Triclosan incorporation on the surface (Figure $3 \mathrm{c}$ and Figure S5a). The nanogel layer is still present and unchanged even when the coating is immersed in $\mathrm{EtOH}$ solution and shaken for $48 \mathrm{~h}$ (Figure S5c).

To determine the loading of the coating with hydrophobic compounds, a technique based on in vivo imaging system (IVIS) fluorescence mapping was applied. IVIS is able to identify the presence of fluorescence, which was introduced by the incorporation of a hydrophobic fluorescent dye into the nanogel coating. Nile Red, a bathochromic dye, was chosen as a hydrophobic dye since it has been frequently used to identify hydrophobic domains within the biomedical field. ${ }^{44,45}$ The fluorescence intensity of Nile Red dramatically increases when it resides within a hydrophobic domain, indicating that when there is high fluorescence intensity, such hydrophobic cavities are present and have been used previously with polymeric micelles. ${ }^{46}$ Thus, the ability to incorporate Triclosan inside the hydrophobic cavities can be proven by a direct comparison of the capability to load Nile Red into the nanogel coatings. Triclosan is a hydrophobic molecule with a similar molecular weight to Nile Red and was previously shown to behave similarly within these particles. ${ }^{3}$

Figure 4 shows the fluorescent images taken by IVIS of the $\mathrm{N}$-nGel and Q-nGel coatings with and without the loading of Nile Red. The N-nGel coating, which is immersed in the Nile Red solution, did not demonstrate any fluorescence signal due to the absence of hydrophobic domains. The Q-nGel coating without Nile Red does not have an appreciable fluorescent

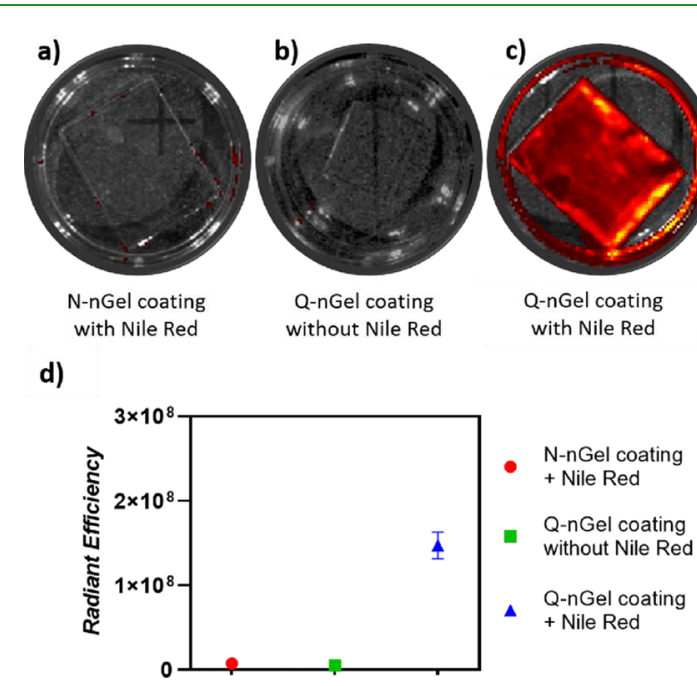

Figure 4. Representative fluorescence images of glass surfaces taken by IVIS: (a) N-nGel coating with Nile Red loading, (b) Q-nGel coating without Nile Red loading, and (c) Q-nGel coating with Nile Red loading. (d) Graph shows a correlation expressed as radiant efficiency $(\mathrm{p} / \mathrm{sec}) /\left(\mu \mathrm{W} / \mathrm{cm}^{2}\right)$ of IVIS imaged surfaces. 
a)

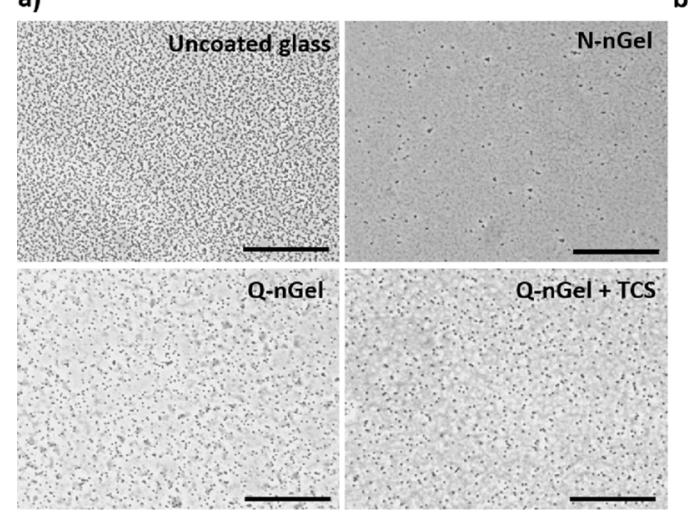

b)



Figure 5. (a) Images of adhered S. aureus ATCC 12600 after $2 \mathrm{~h}$ of flow on uncoated glass, N-nGel coating, Q-nGel coating, and Q-nGel coating +TCS. The scale bar depicts $40 \mu \mathrm{m}$. (b) Number of adhering bacteria after $2 \mathrm{~h}$. Experiments were performed on three independent nanogel-coated surfaces and with separately cultured bacteria. Differences that are statistically significant are marked with $*(\mathrm{p}<0.05), * *(\mathrm{p}<0.01)$, *** $(\mathrm{p}<$ $0.001)$, and $* * * *(\mathrm{p}<0.0001)$.

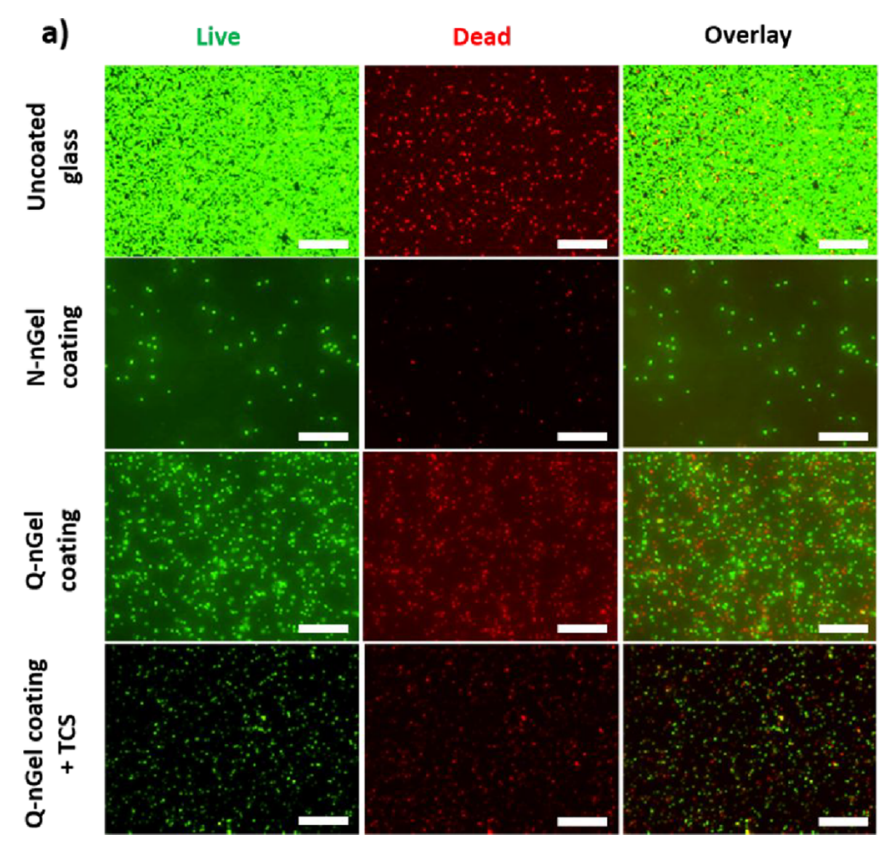

b)

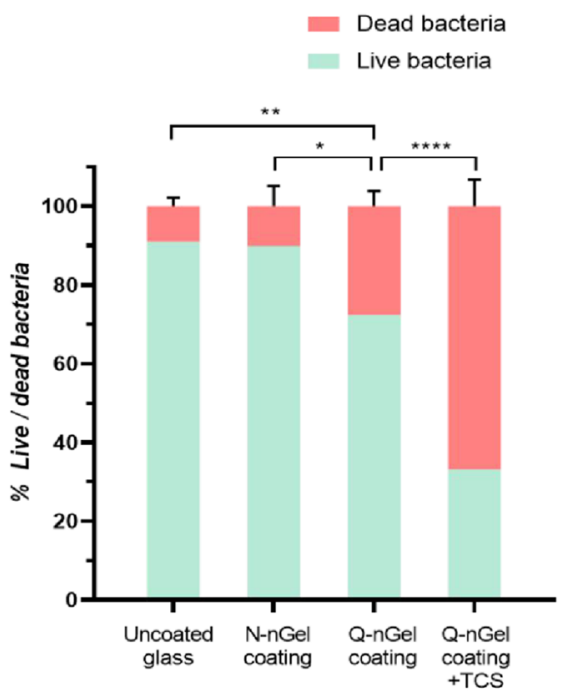

Figure 6. (a) Fluorescence microscopy images of $S$. aureus ATCC 12600 that adhered to glass with and without nanogel coatings after $2 \mathrm{~h}$ under flow. The scale bar depicts $20 \mu \mathrm{m}$. (Intensity has been adjusted for better representation, although for the calculation the originals were used). (b) Adhered bacterial viability was quantified by BacLight LIVE/DEAD staining as a percentage on uncoated glass, N-nGel coating, Q-nGel coating, and Q-nGel coating+TCS. Live and dead bacteria are indicated by green and red, respectively. Experiments were performed on three independent surfaces bearing the nanogel coating and with bacteria that were separately cultured. Differences that are statistically significant are marked with * $(\mathrm{p}<0.05), * *(\mathrm{p}<0.01)$, and $* * * *(\mathrm{p}<0.0001)$.

signal as a control sample, indicating that autofluorescence does not contribute. As can be seen in Figure 4c, upon the introduction of Nile Red, an intense fluorescent signal is present. The representative IVIS images have been quantified, as shown in Figure $4 \mathrm{~d}$, where the Q-nGel with Nile Red displays a radiant efficiency of $1.5 \times 10,{ }^{8}$ which is substantially higher than the other coatings displaying $7.3 \times 10^{6}$ and $4.7 \times$ $10^{6}$ for N-nGel with Nile Red and Q-nGel without Nile Red, respectively.

Antifouling Properties and Antibacterial Activity of Nanogel Coatings. Antifouling Properties. To assess the antifouling properties of the nanogel coatings and to evaluate the differences between the N-nGel coating, Q-nGel coating, and Q-nGel coating+TCS, phase-contrast micrographs of dynamic bacterial adhesion experiments were acquired at the
$2 \mathrm{~h}$ timepoint. Figure 5a qualitatively illustrates the bacterial attachment, which decreases on nanogel-coated surfaces when compared to uncoated glass.

The quantitative results in Figure $5 b$ demonstrate the antifouling properties of the nanogel coatings toward bacterial adhesion. The N-nGel-coated surface shows bacterial adhesion, with a reduction in bacterial adherence of about $97.0 \%$ compared to uncoated glass, which is in line with previously reported data by our group using a different type of nanogel. ${ }^{13}$ When the surface is coated with Q-nGels, the bacterial adhesion increases, and the reduction in adhering bacteria is around $70.9 \%$, which is due to the positively charged QACs of the Q-nGel. The positive charge attracts negatively charged bacteria, but also, the hydrophobic alkyl chains may possibly interact with the membrane, thereby enhancing the 
surface adhesion. Finally, the influence of the Triclosan loading on the antifouling performance of the nanogel coating was quantified as an $86 \%$ reduction of adhering bacteria. It can be stated from these results that adhesion of bacteria are inhibited due to the presence of the hydrated nanogels present at the surface.

Antibacterial Activity of the Nanogel Coatings. To examine the nanogel coatings concerning antibacterial properties, the adhering bacteria were stained with Live/Dead stain after $2 \mathrm{~h}$ under flow and the bacterial viability on the surface was investigated by fluorescence microscopy. As shown in Figure $6 \mathrm{a}, \mathrm{b}$, the $\mathrm{N}$-nGel coating shows similar properties compared to the uncoated glass, as almost $10.1 \%$ of bacteria are dead on the surface, compared to $9.0 \%$ of dead bacteria for the uncoated glass. The N-nGel coating does not show killing properties toward adhered bacteria on the surface; therefore, it can be classified as an antifouling coating rather than being an antibacterial coating, as expected. The results show that the QnGel coating and Q-nGel coating with Triclosan loading present an efficient killing of $S$. aureus bacteria on the surface, 27.6 and $67.0 \%$, respectively. As can be seen, the incorporation of Triclosan almost triples the number of dead bacteria when compared to without Triclosan loading; hence, the loading amplifies the antibacterial activity (Figure 6b). Even though the quaternization of the $\mathrm{N}-\mathrm{nGel}$ reduces the antifouling property of the coating (Figure 5), it still actively kills the bacteria upon adhesion, particularly when the coating is combined with Triclosan that is additionally being delivered into the bacteria upon adherence and enhances the killing efficiency.

In principle, live-dead staining results solely indicate membrane disruption, ${ }^{47}$ while determination of the bacterial cell death is solely possible from culture-based assays. Focusing on the contact killing aspect here rather than the antifouling behavior under flow conditions, we used culture-based Petrifilm assays (Figure S6). As the Q-nGels were sprayed and immobilized onto the glass surface owing to the electrostatic interactions created between the charges of nanogels and the surface, the bacterial lipid membrane is most likely the only part of bacteria with which the nanogels are in contact. Through this contact, the negatively charged bacterium might be attracted by the positively charged nanogels to the surface, which leads to membrane disruption and killing (Table S1). As depicted in Figure S6, the images qualitatively show that Triclosan-loaded $\mathrm{nGel}$ coatings possess an enhanced killing toward bacteria when compared to Q-nGel coatings.

To identify and quantify the antibacterial aspects concerning the nanogel coatings, the colony-forming unit (cfu) counting method, which is another culture-based assay, was carried out. Through the cfu method, only the viable bacteria can be counted and quantified, as this technique excludes dead bacteria.

According to the antibacterial activity assays (Figure 7), also the Q-nGel coating is able to kill S. aureus ATCC 12600 by direct contact; incorporating Triclosan drastically increases the killing efficiency. Particularly, when the bacterial cell membrane opens up by the electrostatic and hydrophobic chain disruption, Triclosan can be released inside the cell membrane and thereby kill the bacterium. Previously we showed that passive diffusion does not liberate Triclosan.

As depicted in Figure 7, the most effective bactericidal killing has been observed by a remarkable decrease (99.99\%) in cfu

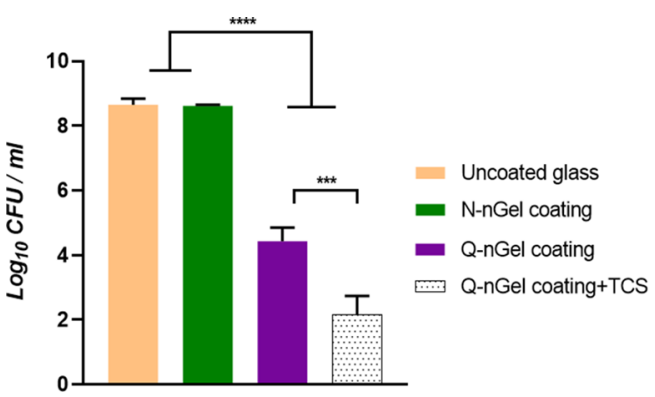

Figure 7. Determined number of colony forming units of surviving $S$. aureus ATCC 12600 after $24 \mathrm{~h}$ incubation on the surface of uncoated glass, N-nGel coating, Q-nGel coating, and Q-nGel coating+TCS. Experiments were performed on three independent surfaces that were coated with nanogels and with bacteria that were cultured separately. Differences that are statistically significant are marked with $* * *(\mathrm{p}<$ $0.001)$ and $* * * *(\mathrm{p}<0.0001)$.

numbers upon the release of Triclosan from the nanogel structure on the coated surface. In comparison to other studies using Triclosan-loaded polymers or micelles that also displayed effective killing, the focus lies on the treatment of the infections ${ }^{48,49}$ while our our nanogel particle coatings show promising results to design an efficient antimicrobial system that prevents infections. This is assigned to a successful collaboration between the hydrophobic chain as it destroys the bacterial membrane and Triclosan as it kills the bacteria.

Furthermore, to identify the absence of unspecific Triclosan release from the nanogel, UV-vis absorption spectroscopy has been performed. Triclosan-incorporated Q-nGel coatings are immersed into the water for different periods as $1,3,6$, and 24 $h$, and in Figure S7a, the UV absorbance spectra of these solutions have been compared. The absorption signal at $\sim 280$ $\mathrm{nm}$ that results from Triclosan, as supported by the UV-vis spectrum of an aqueous solution Triclosan, is not observed even after extensive washing. ${ }^{50}$ In the depicted spectra, no absorption related to Triclosan was detected even at $24 \mathrm{~h}$ from the Triclosan-loaded nanogel coatings. It was further confirmed by using Nile Red, which was still detectable by IVIS even after $24 \mathrm{~h}$ in water when entrapped inside the nanogel coating, proving that the encapsulation is successful and that there is no detectable unspecific release unless there is contact with bacteria (Figure S7b).

\section{CONCLUSIONS}

In this work, a novel quaternized, drug-loaded nanogel system that was successfully translated into surface coatings comprising Triclosan as the antimicrobial agent has been investigated in order to obtain a multifunctional combination of antifouling and bacterial-killing coating properties. First, we have demonstrated a tertiary amine-functionalized nanogel network that could be successfully deposited as a surface coating, and the reduction in the number of adhered bacteria is $97 \%$, as expected from nanogel systems owing to their hydration layer. Next, the quaternization was performed from the tertiary amine groups of these N-nGels using 1-bromododecane, and the surface coatings reveal that the quaternized nanogel coatings have an impact on the bacterial-killing potency. Furthermore, the Triclosan loading is achieved by the hydrophobic interactions in the hydrophobic pockets of the nanogel network, which are created through the intraparticle micellization of the dodecane chain in an aqueous environment. Triclosan release from the nanogel occurs when the 
bacterial lipid membrane interacts with the nanogel allowing Triclosan to enter the bacterial cell. The best results from livedead staining experiments were obtained for the Triclosanloaded surface coatings, which led to a significant enhancement of the antimicrobial activity up to three times compared to $\mathrm{N}$ nGel-coated surfaces. Similar results were obtained when performing Petrifilm assays and the cfu counting method proving that quaternized nanogels disrupted the bacterial cell membrane in the surface-attached states providing Triclosan release to increase the killing activity reaching a $99.99 \%$ efficiency. It should be noted that there is a trade-off between antifouling efficiency and killing capability. Even though the amount of adhered bacteria is lower on the Q-nGel+TCS than on the Q-nGel alone, indicating that killing the bacteria is beneficial for detachment of the bacteria, it is not as effective in repulsion as the $\mathrm{N}$-nGel coating. This delicate balance of effectiveness is common for most contact killing coatings, and even though the coating presented here has a significant reduction, there is still room for improvement.

These results confirm that nanogels provide an exciting approach to prevent bacterial adhesion on the surface but also demonstrate, for the first time, the possibility to achieve Triclosan encapsulation into the nanogel for a powerful antibacterial effect. An appropriate selection of the nanogel functional groups and coating preparation together with antimicrobial drug encapsulation on the coating surface resulted in an excellent performing antimicrobial structure. Future focus will not only entail other hydrophobic therapeutic agents but also include extensive biological screening and testing including hemocompatibility, immune response, complement activation, and in vivo performance. This development is vital for further successful development of antimicrobial-carrying multifunctional nanogel coatings toward future clinically applied coatings.

\section{EXPERIMENTAL SECTION}

Materials. 2,2'-Azobis(2-methylpropioamidine)dihydrochloride (AMPA, V50, 97\%), N-[3-(dimethylamino)propyl]methacrylamide (DMAPMA, 99\%), $N, N^{\prime}$ methylene-bis(acrylamide) (BIS, 99\%), potassium carbonate $\left(\mathrm{K}_{2} \mathrm{CO}_{3}\right)$, Nile Red, hexadecyltrimethylammonium bromide (CTAB, 99\%), N,N-dimethylformamide (DMF, anhydrous), methyl iodide, polyethylenimine (PEI, branched, $\mathrm{Mw}$ $25.000 \mathrm{~g} / \mathrm{mol}$ ), 1-bromo-dodecane (97\%), poly(sodium 4-styrenesulfonate) (PSS, Mw $70.000 \mathrm{~g} / \mathrm{mol})$, and deuterium oxide $\left(\mathrm{D}_{2} \mathrm{O}\right)$ were obtained from Sigma-Aldrich, The Netherlands. $\mathrm{N}$-isopropylacrylamide (NIPAM, 98\%) was obtained from TCI, Belgium. Potassium chloride $(\mathrm{KCl})$, ethanol, methanol (anhydrous), and tetrahydrofuran (THF, anhydrous) were obtained from Merck, Germany. Triclosan was obtained from Duchefa B.V., The Netherlands. All chemicals were used as received without any further purification. Ultrapure water (18.2 $\mathrm{M} \Omega$, arium $611 \mathrm{DI}$ water purification system; Sartorius AG, Göttingen, Germany) was used in all experiments.

Synthesis of the Nonquaternized Nanogel. The N-nGel $\mathrm{P}$ (NIPAM-co-DMAPMA) was prepared via precipitation polymerization. ${ }^{51}$ NIPAM $(3.37 \mathrm{~g})(29.8 \mathrm{mmol}, 85 \mathrm{~mol} \%), 0.27 \mathrm{~g}$ of BIS (1.7 $\mathrm{mmol}, 5 \mathrm{~mol} \%)$, and $0.0106 \mathrm{~g}$ of CTAB (0.03 mmol) were solubilized in ultrapure water $(236 \mathrm{~mL})$ in a three-necked flask of 500 $\mathrm{mL}$, which was equipped with a reflux condenser. Oxygen was removed from the reaction mixture by bubbling $\mathrm{N}_{2}$ through the solution for $1 \mathrm{~h}$. The mixture was heated to $85{ }^{\circ} \mathrm{C}$, and subsequently, $0.6 \mathrm{~g}$ of degassed DMAPMA ( $3.4 \mathrm{mmol}, 10 \mathrm{~mol} \%$ ) was introduced into the solution via a syringe. The $\mathrm{pH}$ was maintained between 8 and 9 using $0.1 \mathrm{M}$ degassed $\mathrm{NaOH}$ and $\mathrm{HCl}$ solutions, and the reaction was initiated by addition of the initiator $(14 \mathrm{~mL}$ degassed aqueous AMPA V50 solution of $0.135 \mathrm{~g}(0.5 \mathrm{mmol}))$ to the reaction. The solution turned turbid after $10 \mathrm{~min}$, and the reaction was continued at
$85{ }^{\circ} \mathrm{C}$ with stirring at $300 \mathrm{rpm}$ under an $\mathrm{N}_{2}$ atmosphere for $6 \mathrm{~h}$. Subsequently, the solution was allowed to cool to room temperature, and stirring was continued overnight. Purification of the nanogel dispersion was done by ultracentrifugation at $179.200 \mathrm{~g}$ and redispersion of the isolated pellet in ultrapure water. The washing sequence was performed 3 times. The P(NIPAM-co-DMAPMA) nGel was isolated by freeze-drying as a powder.

Quaternization of $\mathbf{N}$-nGel to Q-nGel. The previously synthesized N-nGel $(500 \mathrm{mg}$ ) (14 wt \% amine) and $82 \mathrm{mg}$ of $\mathrm{NaOH}$ were dissolved in $100 \mathrm{~mL}$ of DMF in a round-bottom flask equipped with a stirrer. The reaction was started by injection of 1027 $\mathrm{g}$ of 1-bromo-dodecane (BDD). The reaction was stirred for 4 days at $80{ }^{\circ} \mathrm{C}$. Impurities were removed via centrifugation at $12.300 \mathrm{~g}$. After the centrifugation, dialysis (MWCO $3500 \mathrm{kDa}$ ) was performed for 3 days against $96 \%$ ethanol and afterward against water also for 3 days. The pure Q-nGel was isolated as a powder by freeze-drying.

Surface Preparation and Nanogel Coating. A glass substrate was cleaned using $70 \%$ ethanol and water with subsequent drying. Surface activation was done for $10 \mathrm{~min}$ using plasma oxidation at 100 mTorr and 0.2 mbar (Plasma Active Flecto 10 USB). After surface activation, $\mathrm{N}$-nGel suspension $(5 \mathrm{mg} / \mathrm{mL}, 0.5 \mathrm{wt} \%)$ was deposited by spraying it onto the surface of the glass slide till complete surface wetting was achieved ( $8-12$ times) to form the coating on the surface. For the Q-nGel coating, the glass slide was dipped in $1.5 \mathrm{mg} /$ $\mathrm{mL}$ PEI solution for $20 \mathrm{~min}(\mathrm{pH}$ was maintained at $\mathrm{pH} 7$ using an aqueous solution of $0.1 \mathrm{M} \mathrm{HCl}$ ) after plasma oxidation treatment. A PEI-coated glass slide was cleaned three times using ultrapure water and immersed for $20 \mathrm{~min}$ in a $3.0 \mathrm{mg} / \mathrm{mL}(0.3 \mathrm{wt} \%)$ poly(sodium 4styrenesulfonate) (PSS) solution. After rinsing with water and drying at room temperature, Q-nGel suspension $(5 \mathrm{mg} / \mathrm{mL}, 0.5 \mathrm{wt} \%)$ was deposited via spraying on the PSS-coated surface identically. The coated surfaces were initially dried at room temperature and subsequently at $50{ }^{\circ} \mathrm{C}$ overnight. The glass substrates were washed for $6 \mathrm{~h}$ in water, which was refreshed three times. All nanogel coatings were sterilized before use by dipping in $70 \%$ ethanol for $1 \mathrm{~min}$ and afterward washed excessively using sterilized ultrapure water prior to use for the microbiology experiments.

Loading of Nile Red and Triclosan on the Q-nGel Coatings. Nile Red $(10 \mathrm{mg})(0.031 \mathrm{mmol})$ and $(0.035 \mathrm{mmol})$ Triclosan are dissolved in $1 \mathrm{~mL}$ of THF. From this mixture, $5 \mu \mathrm{L}$ is taken and added in $1 \mathrm{~mL}$ of $70 \%$ ethanol. Afterward, $5 \mu \mathrm{L}$ of Nile Red/ethanol and Triclosan/ethanol is added in $5 \mathrm{~mL}$ of ultrapure water. A Q-nGelcoated glass slide $(10 \times 10 \times 1 \mathrm{~mm})$ was then immersed in this mixture, which was continuously shaken at a speed of $50 \mathrm{rpm}$ for $3 \mathrm{~h}$. The Nile Red and Triclosan-loaded nanogel coatings were washed overnight in ultrapure water and dried at ambient temperature before using for further analysis.

Bacterial Strain and Growth Conditions. S. aureus ATCC 12600 strain was grown at $37^{\circ} \mathrm{C}$ overnight on an blood agar from a frozen stock. A single colony inoculated in $10 \mathrm{~mL}$ of tryptone soy broth (TSB; Oxoid, Basingstoke, UK) and the mentioned preculture was incubated for $24 \mathrm{~h}$ at $37^{\circ} \mathrm{C}$ and further used for inoculation of a $200 \mathrm{~mL}$ TSB main culture that was allowed to grow for $16 \mathrm{~h}$. The bacteria were isolated from the second culture via centrifugation at $5000 \mathrm{~g}$ at $10{ }^{\circ} \mathrm{C}$ for $5 \mathrm{~min}$ and washed with potassium phosphatebuffered saline (PBS, $10 \mathrm{mM}$ potassium phosphate, $0.15 \mathrm{M} \mathrm{NaCl}, \mathrm{pH}$ 7.0). Subsequently, to break bacterial clusters and obtain single bacteria, these were sonicated for $30 \mathrm{~s}$ at $30 \mathrm{~W}$ (Vibra Cell model VCX130; Sonics and Materials Inc., Newtown, Connecticut, USA) on ice. Next, the bacterial number in suspension was determined using a Bürker-Türk counting chamber, and for further experiments, the concentration was adjusted accordingly.

Assessment of Bacterial Adhesion. Adhesion of bacteria on the nanogel coatings on glass was done with a custom-built parallel plate flow chamber and by passing the bacterial suspension of $3 \times 10^{8} \mathrm{~mL}^{-1}$ for $2 \mathrm{~h}$ at ambient temperature at a shear rate of $12 \mathrm{~s}^{-1}$, as reported before. $^{52,53}$ Through the flow chamber system, PBS was circulated to remove bubbles before starting each experiment. The size of the area for analysis of the flow chamber was $17 \mathrm{~mm}$ (width) by $67 \mathrm{~mm}$ (length) by $0.75 \mathrm{~mm}$ (height). After $2 \mathrm{~h}$, the flow was terminated and 
replaced for PBS buffer solution for $30 \mathrm{~min}$ at a similar flow rate to get rid of nonattached bacteria.

Enumeration of Live and Dead Staphylococci. The nanogelcoated glass slides were taken from the $2 \mathrm{~h}$ bacterial adhesion experiment immediately and used to assess live and dead bacteria. SYTO 9 and propidium iodide (Molecular Probes, Leiden, The Netherlands) with a 1:1 ratio were mixed for the live/dead staining solution. After addition of the staining solution, samples were incubated for $15 \mathrm{~min}$ at ambient temperature avoiding light exposure. Imaging of the bacteria was performed by fluorescence microscopy (Leica DFC350 FX) (40× plan apochromatic objective) equipped with a highly light-sensitive digital CCD camera and an automatic light shutter. The density of live and dead bacteria was determined using ImageJ and provided as the percentage of red-fluorescence over the sum of red-and green-fluorescent pixels using five different pictures.

Antibacterial Activity of Nanogel Coatings on Biofilm Formation - Colony Count Method. Bacterial suspension (1 $\mathrm{mL})$ in PBS with a concentration of $1.0 \times 10^{6}$ bacteria $/ \mathrm{mL}$ was applied to the coating and incubated for $1 \mathrm{~h}$ at $37^{\circ} \mathrm{C}$ to allow for adherence of the bacteria. Subsequently, the PBS was taken out carefully and the slides were immersed into $1 \mathrm{~mL}$ of TSB growth medium, and bacteria were cultured on the coatings at $37^{\circ} \mathrm{C}$ for $24 \mathrm{~h}$. After incubation, the coatings were washed three times with PBS to remove the poorly attached bacteria. Coatings were subjected to an ultrasonic bath in $1 \mathrm{~mL}$ of PBS for $5 \mathrm{~min}$ in order to remove the biofilm that had developed on the coating surface. The detached bacterial suspension was diluted serially in 10-fold steps with PBS. The dilute suspensions were plated on a TSB agar plate and incubated overnight at $37^{\circ} \mathrm{C}$, and subsequently, the colony-forming units per $\mathrm{ml}$ $(\mathrm{cfu} / \mathrm{mL})$ were identified.

Contact-Killing Activity of Nanogel Coatings. To evaluate the contact-killing potential of nanogel coatings, the Petrifilm Aerobic Count plate system ( $3 \mathrm{M}$ Microbiology, USA) was applied. Petrifilm plates consisted of two foil pieces containing nutrients, a cold-watersoluble gel, and a tetrazolium indicator for easy identification of the number of colonies on top of the sample. These plates were prepared $1 \mathrm{~h}$ before use by hydrating the gel with $1 \mathrm{~mL}$ of sterilized ultrapure water. Then, the coated substrate was placed in between the two foil pieces and placed together with $15 \mathrm{~mL}$ of the overnight inoculum (3 $\times 10^{5}$ bacteria $/ \mathrm{ml}$ ) and the gel, to ensure that the bacterial suspension is homogeneously distributed over the whole sample. The films were incubated for $48 \mathrm{~h}$ at $37^{\circ} \mathrm{C}$. The coating was positively assessed for contact killing in the absence of colonies in contact with the gel, while the presence of colonies indicated negative for contact killing.

Characterization Methods. The hydrodynamic radius $\left(R_{\mathrm{h}}\right)$, size distributions, and zeta potentials of the nanogels were identified using a Zetasizer Nano-ZS (Malvern Instruments, Worcestershire, UK) in ultrapure water. Temperature-dependent measurements were logged at a fixed scattering angle of $173^{\circ}$ and a wavelength $\lambda=633 \mathrm{~nm}$ of the laser beam, while variations in temperature were used in the range of 20 to $50{ }^{\circ} \mathrm{C}$ at $2{ }^{\circ} \mathrm{C}$ intervals and with $10 \mathrm{~s}$ measurement time and 11 runs and performed in triplicate. Zeta potential measurements were performed in water using capillary cells (Malvern, DTS1070) that are disposable at an angle of $17^{\circ}$ and a laser beam wavelength of $\lambda=633$ $\mathrm{nm}$.

TEM analysis was carried out using a Phillips CM12 microscope operating at an accelerating voltage of $120 \mathrm{kV}$ and coupled to a $4 \mathrm{k}$ CCD camera. Uranium acetate was used to stain the samples that were prepared by drop-casting of a nanogel suspension $(0.5 \mathrm{mg} / \mathrm{mL})$ onto a copper grid modified with carbon.

A Varian Mercury-400 NMR spectrometer $(400 \mathrm{MHz})$ was used for nuclear magnetic resonance. All spectra were taken at ambient temperature. $\mathrm{D}_{2} \mathrm{O}$ was used as a solvent, and a nanogel concentration of $10 \mathrm{mg} / \mathrm{mL}$ was used. The proton signal of residual $\mathrm{D}_{2} \mathrm{O}$ was used as a reference.

The surface morphology of the nanogel coatings was observed with AFM (Dimension 3100 Nanoscope V, Veeco, Plainview, NY, USA) in contact mode using DNP cantilevers (spring constant $\mathrm{k}=0.06 \mathrm{~N} / \mathrm{m}$ or $\mathrm{k}=0.24 \mathrm{~N} / \mathrm{m}$ and resonant frequency $\mathrm{f}_{0}=18 \mathrm{kHz}$ or $\mathrm{f}_{0}=56 \mathrm{kHz}$ ) made from silicon nitride in the dry state.

The IVIS Lumina II, Imaging System (PerkinElmer), a bio-optical Imaging System, was used to detect the fluorescence signal on the nanogel coating. Fluorescence images were taken and adjusted automatically for background signals. Three independent regions of interest (ROIs) (each $0.15 \mathrm{~cm}^{2}$ ) were created manually for every surface, and average radiances for these ROIs were transformed into radiant efficiency $\left((\mathrm{p} / \mathrm{sec}) /\left(\mu \mathrm{W} / \mathrm{cm}^{2}\right)\right)$ using Living Image software (PerkinElmer).

The Triclosan loading of Q-nGel coating + TCS was determined by UV-vis absorption measurements. The nanogel-coated surface was suspended in $5 \mathrm{~mL}$ of water and was placed on a shaker at 120 $\mathrm{rpm}$. One microliter aliquots were taken out of the dissolution suspension at exact time points $(1,3,6$, and $24 \mathrm{~h})$ and replaced by the same volume of freshwater, to keep the volume of the release solution constant. For comparison reasons, a Triclosan solution in water (5 $\mu \mathrm{g} / \mathrm{mL}$ ) was prepared. The absorption at $280 \mathrm{~nm}$ of the nanogel solution was measured using a UV-vis spectrophotometer (Perkin Elmer Lambda $2 \mathrm{~s}$ ).

A phase-contrast microscope (OlympusBH-2) was used to monitor bacterial adhesion, and live images $(1392 \times 1040$ pixels with 8 -bit resolution) were taken after the summation of 15 consecutive images (time interval $1 \mathrm{~s}$ ) to enhance the signal to noise ratio and eliminate moving bacteria from the analysis. The bacteria that were individually adsorbed were determined within five images, which were taken at various positions on the substrates after $2 \mathrm{~h}$ bacterial adhesion. The total number of individual surface attached bacteria was enumerated from the obtained images in both a manual approach and by using software. The number of bacteria that adhered per $\mathrm{cm}^{2}$ was counted using software that was developed in-house and is based on MATLAB. If more than ca. 300 bacteria were present, the software was used, while a manual approach was used when the number was well below 300 per image. The bacterial density was determined.

All values associated with the presented experiments are averages of three independently fabricated surfaces (coated and uncoated) and conducted with bacteria that were separately cultured. Statistical analysis is performed using a one-way analysis of variance (ANOVA) followed by Tukey's test. At a value of $p<0.05$, it is considered statistical significant.

\section{ASSOCIATED CONTENT}

\section{Supporting Information}

The Supporting Information is available free of charge at https://pubs.acs.org/doi/10.1021/acsami.0c18172.

(Figure S1) N-nGel synthesis and the quaternization reaction to Q-nGel, (Figure S2) ${ }^{1} \mathrm{H}-\mathrm{NMR}$ spectrum corresponding to N-nGel and Q-nGel, (Figure S3) hydrodynamic radius $\left(R_{\mathrm{h}}\right)$ as a function of the temperature of $\mathrm{N}-\mathrm{nGel}$ and Q-nGel, (Figure S4) AFM images of (a) Q-nGel coating on the plasma-oxidized glass surface, (b) PEI and subsequent PSS coating on the plasma-oxidized glass surface at $23{ }^{\circ} \mathrm{C}$ in the dry state, (Figure S5) Triclosan incorporation and AFM images of loaded Q-nGel coating after washing, (Figure S6) Petrifilm plate images of S. aureus ATCC 12600 after a $48 \mathrm{~h}$ incubation on the surface of uncoated glass, N-nGel coating, Q-nGel coating, and Q-nGel coating +TCS, (Table S1) contact killing capability of the nanogel coatings using the Petrifilm system, and (Figure S7) Triclosan loading and stability of confinement analysis (PDF) 


\section{AUTHOR INFORMATION}

\section{Corresponding Author}

Patrick van Rijn - University of Groningen and University Medical Center Groningen, Department of Biomedical Engineering, W. J. Kolff Institute for Biomedical Engineering and Materials Science, 9713 AV Groningen, The Netherlands; ๑ orcid.org/0000-0002-2208-5725; Email: p.van.rijn@umcg.nl

\section{Authors}

Damla Keskin - University of Groningen and University Medical Center Groningen, Department of Biomedical Engineering, W. J. Kolff Institute for Biomedical Engineering and Materials Science, 9713 AV Groningen, The Netherlands

Lisa Tromp - University of Groningen and University Medical Center Groningen, Department of Biomedical Engineering, W. J. Kolff Institute for Biomedical Engineering and Materials Science, 9713 AV Groningen, The Netherlands

Olga Mergel - University of Groningen and University Medical Center Groningen, Department of Biomedical Engineering, W. J. Kolff Institute for Biomedical Engineering and Materials Science, 9713 AV Groningen, The Netherlands

Guangyue $\mathbf{Z u}$ - University of Groningen and University Medical Center Groningen, Department of Biomedical Engineering, W. J. Kolff Institute for Biomedical Engineering and Materials Science, 9713 AV Groningen, The Netherlands; ๑ orcid.org/0000-0001-5252-8876

Eliza Warszawik - University of Groningen and University Medical Center Groningen, Department of Biomedical Engineering, W. J. Kolff Institute for Biomedical Engineering and Materials Science, 9713 AV Groningen, The Netherlands

Henny C. van der Mei - University of Groningen and University Medical Center Groningen, Department of Biomedical Engineering, W. J. Kolff Institute for Biomedical Engineering and Materials Science, 9713 AV Groningen, The Netherlands; ๑ orcid.org/0000-0003-0760-8900

Complete contact information is available at: https://pubs.acs.org/10.1021/acsami.0c18172

\section{Author Contributions}

The manuscript was written through contributions of all authors. All authors have given approval to the final version of the manuscript.

\section{Funding}

The authors acknowledge the financial support of the European Union's Horizon 2020 research and innovation program under the Marie Skłodowska-Curie grant agreement No 713482 (ALERT Cofound) and the China Scholarship Council (CSC; G.Z. no. 201706890012); O.M. was funded by the Alexander von Humboldt Foundation for the Feodor Lynen Research Fellowship; E.M.W. was funded by TKI Health Holland.

\section{Notes}

The authors declare the following competing financial interest(s): P.v.R also is co-founder, scientific advisor, and share-holder of BiomACS BV, a biomedical oriented screening company. The authors declare no other competing interests.

\section{ACKNOWLEDGMENTS}

The manuscript was written through the contributions of all authors. All authors have approved the final version of the manuscript. The authors thank Reinier Bron and Willem Woudstra for the helpful discussions regarding biology experiments.

\section{REFERENCES}

(1) Arciola, C. R.; Campoccia, D.; Montanaro, L. Implant Infections: Adhesion, Biofilm Formation and Immune Evasion. Nat. Rev. Microbiol. 2018, 16, 397-409.

(2) Magill, S. S.; Edwards, J. R.; Bamberg, W.; Beldavs, Z. G.; Dumyati, G.; Kainer, M. A.; Lynfield, R.; Maloney, M.; McAllisterHollod, L.; Nadle, J.; Ray, S. M.; Thompson, D. L.; Wilson, L. E.; Fridkin, S. K. Multistate Point-Prevalence Survey of Health CareAssociated Infections. N. Engl. J. Med. 2014, 370, 1198-1208.

(3) Jamal, M.; Ahmad, W.; Andleeb, S.; Jalil, F.; Imran, M.; Nawaz, M. A.; Hussain, T.; Ali, M.; Rafiq, M.; Kamil, M. A. Bacterial Biofilm and Associated Infections. J. Chin. Med. Assoc. 2018, 81, 7-11.

(4) Flemming, H.-C.; Wingender, J. The Biofilm Matrix. Nat. Rev. Microbiol. 2010, 8, 623-633.

(5) Hou, J.; Veeregowda, D. H.; van de Belt-Gritter, B.; Busscher, H. J.; van der Mei, H. C. Extracellular Polymeric Matrix Production and Relaxation under Fluid Shear and Mechanical Pressure in \&lt;Span Class=\&quot;Named-Content Genus-Species\&quot; Id=\&quot;Named-Content-1\&quot;\&gt;Staphylococcus Aureus\&lt;/Span\&gt; Biofilms. Appl. Environ. Microbiol. 2018, 84, e01516-e01517.

(6) Harro, J. M.; Peters, B. M.; O’May, G. A.; Archer, N.; Kerns, P.; Prabhakara, R.; Shirtliff, M. E. Vaccine Development in Staphylococcus Aureus: Taking the Biofilm Phenotype into Consideration. FEMS Immunol. Med. Microbiol. 2010, 59, 306-323.

(7) Kolodkin-Gal, I.; Romero, D.; Cao, S.; Clardy, J.; Kolter, R.; Losick, R. \&lt;Span Class=\&quot;Sc\&quot;\&gt;D\&lt;/Span\&gt;Amino Acids Trigger Biofilm Disassembly. Science 2010, 328, 627629.

(8) Bridier, A.; Briandet, R.; Thomas, V.; Dubois-Brissonnet, F. Resistance of Bacterial Biofilms to Disinfectants: A Review. Biofouling 2011, 27, 1017-1032.

(9) Nadell, C. D.; Drescher, K.; Wingreen, N. S.; Bassler, B. L. Extracellular Matrix Structure Governs Invasion Resistance in Bacterial Biofilms. ISME J. 2015, 9, 1700-1709.

(10) Zander, Z. K.; Becker, M. L. Antimicrobial and Antifouling Strategies for Polymeric Medical Devices. ACS Macro Lett. 2018, 7, $16-25$.

(11) Saxena, S.; Spears, M. W., Jr.; Yoshida, H.; Gaulding, J. C.; García, A. J.; Lyon, L. A. Microgel Film Dynamics Modulate Cell Adhesion Behavior. Soft Matter 2014, 10, 1356-1364.

(12) Sigolaeva, L. V.; Gladyr, S. Y.; Gelissen, A. P. H.; Mergel, O.; Pergushov, D. V.; Kurochkin, I. N.; Plamper, F. A.; Richtering, W. Dual-Stimuli-Sensitive Microgels as a Tool for Stimulated Spongelike Adsorption of Biomaterials for Biosensor Applications. Biomacromolecules 2014, 15, 3735-3745.

(13) Keskin, D.; Mergel, O.; van der Mei, H. C.; Busscher, H. J.; van Rijn, P. Inhibiting Bacterial Adhesion by Mechanically Modulated Microgel Coatings. Biomacromolecules 2019, 20, 243-253.

(14) Nyström, L.; Nordström, R.; Bramhill, J.; Saunders, B. R.; Álvarez-Asencio, R.; Rutland, M. W.; Malmsten, M. Factors Affecting Peptide Interactions with Surface-Bound Microgels. Biomacromolecules 2016, 17, 669-678.

(15) Kujur, A.; Kiran, S.; Dubey, N. K.; Prakash, B. Microencapsulation of Gaultheria Procumbens Essential Oil Using Chitosan-Cinnamic Acid Microgel: Improvement of Antimicrobial Activity, Stability and Mode of Action. LWT 2017, 86, 132-138.

(16) Rose, J. C.; Gehlen, D. B.; Haraszti, T.; Köhler, J.; Licht, C. J.; De Laporte, L. Biofunctionalized Aligned Microgels Provide 3D Cell Guidance to Mimic Complex Tissue Matrices. Biomaterials 2018, 163, $128-141$. 
(17) Singh, N.; Bridges, A. W.; García, A. J.; Lyon, L. A. Covalent Tethering of Functional Microgel Films onto Poly(Ethylene Terephthalate) Surfaces. Biomacromolecules 2007, 8, 3271-3275.

(18) Uhlig, K.; Wegener, T.; He, J.; Zeiser, M.; Bookhold, J.; Dewald, I.; Godino, N.; Jaeger, M.; Hellweg, T.; Fery, A.; Duschl, C. Patterned Thermoresponsive Microgel Coatings for Noninvasive Processing of Adherent Cells. Biomacromolecules 2016, 17, 11101116.

(19) Wei, J.; Cai, J.; Li, Y.; Wu, B.; Gong, X.; Ngai, T. Investigation of Cell Behaviors on Thermo-Responsive PNIPAM Microgel Films. Colloids Surf. B: Biointerfaces 2015, 132, 202-207.

(20) Liu, Y.; Xing, L.; Zhang, Q.; Mu, Q.; Liu, P.; Chen, K.; Chen, L.; Zhang, X.; Wang, K.; Wei, Y. Thermo- and Salt-Responsive Poly(NIPAm-Co-AAc-Brij-58) Microgels: Adjustable Size, Stability under Salt Stimulus, and Rapid Protein Adsorption/Desorption. Colloid Polym. Sci. 2016, 294, 617-628.

(21) Saha, P.; Santi, M.; Frenken, M.; Palanisamy, A. R.; Ganguly, R.; Singha, N. K.; Pich, A. Dual-Temperature-Responsive Microgels from a Zwitterionic Functional Graft Copolymer with Superior Protein Repelling Property. ACS Macro Lett. 2020, 9, 895-901.

(22) Brosel-Oliu, S.; Mergel, O.; Uria, N.; Abramova, N.; van Rijn, P.; Bratov, A. 3D Impedimetric Sensors as a Tool for Monitoring Bacterial Response to Antibiotics. Lab Chip 2019, 19, 1436-1447.

(23) Nolan, C. M.; Reyes, C. D.; Debord, J. D.; García, A. J.; Lyon, L. A. Phase Transition Behavior, Protein Adsorption, and Cell Adhesion Resistance of Poly(Ethylene Glycol) Cross-Linked Microgel Particles. Biomacromolecules 2005, 6, 2032-2039.

(24) South, A. B.; Whitmire, R. E.; García, A. J.; Lyon, L. A. Centrifugal Deposition of Microgels for the Rapid Assembly of Nonfouling Thin Films. ACS Appl. Mater. Interfaces 2009, 1, 27472754.

(25) Vatankhah-Varnosfaderani, M.; Hu, X.; Li, Q.; Adelnia, H.; Ina, M.; Sheiko, S. S. Universal Coatings Based on ZwitterionicDopamine Copolymer Microgels. ACS Appl. Mater. Interfaces 2018, 10, 20869-20875.

(26) Sigolaeva, L. V.; Pergushov, D. V.; Oelmann, M.; Schwarz, S.; Brugnoni, M.; Kurochkin, I. N.; Plamper, F. A.; Fery, A.; Richtering, W. Surface Functionalization by Stimuli-Sensitive Microgels for Effective Enzyme Uptake and Rational Design of Biosensor Setups. Polymers 2018, 10, 791.

(27) Lynch, I.; Miller, I.; Gallagher, W. M.; Dawson, K. A. Novel Method to Prepare Morphologically Rich Polymeric Surfaces for Biomedical Applications via Phase Separation and Arrest of Microgel Particles. J. Phys. Chem. B 2006, 110, 14581-14589.

(28) Xia, Y.; Tang, D.; Wu, H.; Wang, X.; Cao, M.; He, H.; Wang, S. Cell Attachment/Detachment Behavior on Poly(N-Isopropylacrylamide)-Based Microgel Films: The Effect of Microgel Structure and Swelling Ratio. J. Mater. Sci. 2018, 53, 8795-8806.

(29) Nyström, L.; Strömstedt, A. A.; Schmidtchen, A.; Malmsten, M. Peptide-Loaded Microgels as Antimicrobial and Anti-Inflammatory Surface Coatings. Biomacromolecules 2018, 19, 3456-3466.

(30) Schmidt, S.; Zeiser, M.; Hellweg, T.; Duschl, C.; Fery, A.; Möhwald, H. Adhesion and Mechanical Properties of PNIPAM Microgel Films and Their Potential Use as Switchable Cell Culture Substrates. Adv. Funct. Mater. 2010, 20, 3235-3243.

(31) Schmidt, S.; Hellweg, T.; von Klitzing, R. Packing Density Control in P(NIPAM-Co-AAc) Microgel Monolayers: Effect of Surface Charge, PH, and Preparation Technique. Langmuir 2008, 24, 12595-12602.

(32) Wang, Q.; Uzunoglu, E.; Wu, Y.; Libera, M. Self-Assembled Poly(Ethylene Glycol)- Co-Acrylic Acid Microgels to Inhibit Bacterial Colonization of Synthetic Surfaces. ACS Appl. Mater. Interfaces 2012, 4, 2498-2506.

(33) Mergel, O.; Schneider, S.; Tiwari, R.; Kühn, P. T.; Keskin, D.; Stuart, M. C. A.; Schöttner, S.; de Kanter, M.; Noyong, M.; Caumanns, T.; Mayer, J.; Janzen, C.; Simon, U.; Gallei, M.; Wöll, D.; van Rijn, P.; Plamper, F. A. Cargo Shuttling by Electrochemical Switching of Core-Shell Microgels Obtained by a Facile One-Shot Polymerization. Chem. Sci. 2019, 10, 1844-1856.
(34) Ping, M.; Zhang, X.; Liu, M.; Wu, Z.; Wang, Z. Surface Modification of Polyvinylidene Fluoride Membrane by Atom-Transfer Radical-Polymerization of Quaternary Ammonium Compound for Mitigating Biofouling. J. Membr. Sci. 2019, 570-571, 286-293.

(35) Zhang, X.; Ma, J.; Tang, C. Y.; Wang, Z.; Ng, H. Y.; Wu, Z. Antibiofouling Polyvinylidene Fluoride Membrane Modified by Quaternary Ammonium Compound: Direct Contact-Killing versus Induced Indirect Contact-Killing. Environ. Sci. Technol. 2016, 50, 5086-5093.

(36) Dong, J. J.; Muszanska, A.; Xiang, F.; Falkenberg, R.; van de Belt-Gritter, B.; Loontjens, T. Contact Killing of Gram-Positive and Gram-Negative Bacteria on PDMS Provided with Immobilized Hyperbranched Antibacterial Coatings. Langmuir 2019, 35, 1410814116.

(37) Zu, G.; Steinmuller, M.; Keskin, D.; van der Mei, H. C.; Mergel, O.; van Rijn, P. Antimicrobial Nanogels with Nano-Injection Capabilities for Delivery of Hydrophobic Antibacterial Agent Triclosan. ACS Appl. Polym. Mater. 2020, DOI: 10.1021/acsapm.0c01031.

(38) Monteux, C.; Marlière, C.; Paris, P.; Pantoustier, N.; Sanson, N.; Perrin, P. Poly(N-Isopropylacrylamide) Microgels at the OilWater Interface: Interfacial Properties as a Function of Temperature. Langmuir 2010, 26, 13839-13846.

(39) Müller, M.; Urban, B.; Reis, B.; Yu, X.; Grab, A. L.; CavalcantiAdam, E. A.; Kuckling, D. Switchable Release of Bone Morphogenetic Protein from Thermoresponsive Poly(NIPAM-Co-DMAEMA)/Cellulose Sulfate Particle Coatings. Polymers 2018, 10, 1314.

(40) Tsuji, S.; Kawaguchi, H. Self-Assembly of Poly(N-Isopropylacrylamide)-Carrying Microspheres into Two-Dimensional Colloidal Arrays. Langmuir 2005, 21, 2434-2437.

(41) Schmidt, S.; Motschmann, H.; Hellweg, T.; von Klitzing, R. Thermoresponsive Surfaces by Spin-Coating of PNIPAM-Co-PAA Microgels: A Combined AFM and Ellipsometry Study. Polymer 2008, 49, 749-756

(42) Mani, P.; Pandey, A. K.; Tripathi, A. K. Synthesis and Binding Affinity of Hydrophobic Tail Containing Naphthalene Derivatives with Different Type of Organized Media. ChemistrySelect 2020, 5, 2135-2141.

(43) Lundin, J. G.; Giles, S. L.; Fulmer, P. A.; Wynne, J. H. Distribution of Quaternary Ammonium Salt Encapsulated Polyoxometalates in Polyurethane Films. Prog. Org. Coatings 2017, 105, $320-329$.

(44) Rumin, J.; Bonnefond, H.; Saint-Jean, B.; Rouxel, C.; Sciandra, A.; Bernard, O.; Cadoret, J.-P.; Bougaran, G. The Use of Fluorescent Nile Red and BODIPY for Lipid Measurement in Microalgae. Biotechnol. Biofuels 2015, 8, 42.

(45) Maes, T.; Jessop, R.; Wellner, N.; Haupt, K.; Mayes, A. G. A Rapid-Screening Approach to Detect and Quantify Microplastics Based on Fluorescent Tagging with Nile Red. Sci. Rep. 2017, 7, 44501.

(46) Trimaille, T.; Mondon, K.; Gurny, R.; Moller, M. Novel Polymeric Micelles for Hydrophobic Drug Delivery Based on Biodegradable Poly(Hexyl-Substituted Lactides). Int. J. Pharm. 2006, 319, 147-154.

(47) Stocks, S. M. Mechanism and Use of the Commercially Available Viability Stain. BacLight. Cytom. Part A 2004, 61A, 189195.

(48) Liu, Y.; Busscher, H. J.; Zhao, B.; Li, Y.; Zhang, Z.; van der Mei, H. C.; Ren, Y.; Shi, L. Surface-Adaptive, Antimicrobially Loaded, Micellar Nanocarriers with Enhanced Penetration and Killing Efficiency in Staphylococcal Biofilms. ACS Nano 2016, 10, 47794789.

(49) Su, Y.; Zhao, L.; Meng, F.; Qiao, Z.; Yao, Y.; Luo, J. Triclosan Loaded Polyurethane Micelles with $\mathrm{PH}$ and Lipase Sensitive Properties for Antibacterial Applications and Treatment of Biofilms. Mater. Sci. Eng. C 2018, 93, 921-930.

(50) Wong-Wah-Chung, P.; Rafqah, S.; Voyard, G.; Sarakha, M. Photochemical Behaviour of Triclosan in Aqueous Solutions: Kinetic and Analytical Studies. J. Photochem. Photobiol., A 2007, 191, 201208. 
(51) Mergel, O.; Gelissen, A. P. H.; Wünnemann, P.; Böker, A.; Simon, U.; Plamper, F. A. Selective Packaging of Ferricyanide within Thermoresponsive Microgels. J. Phys. Chem. C 2014, 118, 2619926211.

(52) Roosjen, A.; van der Mei, H. C.; Busscher, H. J.; Norde, W. Microbial Adhesion to Poly(Ethylene Oxide) Brushes: Influence of Polymer Chain Length and Temperature. Langmuir 2004, 20, 1094910955.

(53) Busscher, H. J.; van der Mei, H. C. Microbial Adhesion in Flow Displacement Systems. Clin. Microbiol. Rev. 2006, 19, 127-141. 